\title{
Análisis tribológico en un motor de gasolina con dos marcas de lubricantes y la misma especificación
}

\section{Análisis tribológico en un motor de gasolina con dos marcas de lubricantes y la misma especificación}

\author{
Abel Polivio Remache Coyago \\ Universidad Central del Ecuador, Ecuador \\ Edwin Giovanny Puente Moromenacho \\ Ricardo David Aulestia Martínez \\ Marco Vinicio Noroña Merchán \\ Universidad Internacional del Ecuador, Ecuador \\ Autor para correspondencia: abelbelo@hotmail.com, epuente@internacional.edu.ec, \\ ricardoamd9@gmail.com, manorame@internacional.edu.ec \\ Fecha de recepción: 3 de enero de 2017 - Fecha de aceptación: 20 de Febrero de 2017
}

Resumen: En la actualidad el lubricante de un motor para automóvil no es tomado con la importancia del caso, haciendo que los consumidores le den mayor importancia al precio del mismo antes que a su calidad, y componentes. La investigación realizó un análisis de aceites a través de una prueba tribológica en un motor de gasolina, con el objetivo de conocer el desgaste de los componentes internos del motor, basándose en los límites condenatorios y sus rangos establecidos. Se utilizó dos marcas de lubricantes tipo SAE 20W50, API SN, para realizar un cuadro comparativo entre ellos y bajo las mismas condiciones de trabajo. Entre los resultados se observó que el aceite A generaba mayor porcentaje de hollín: 0,02\% superior al B, por razones de viscosidad inferior a alta temperatura. Se encontró una sustancial diferencia, en cantidad de oxidación por un valor superior al $27 \%$ en el aceite B, lo que afecta específicamente a la vida útil del lubricante y pone en riesgo la protección de los componentes del motor. Las cantidades de $\mathrm{Cu}$, Si y Al se encontraban dentro de los límites condenatorios tanto en el lubricante A como en el lubricante B la diferencia no fue sustancial es estos productos. En cambio los valores de $\mathrm{Na}$ estuvieron fuera del rango (23 y $24 \mathrm{ppm}$ ) por fugas de líquido refrigerante. Se da a conocer entonces que a pesar de tener una misma especificación entre diferentes marcas, se pueden generar diferentes degastes debido a los componentes internos y características tipo de cada aceite.

Palabras Clave: tribología; desgaste de motores; aceites; lubricantes

Abstract: At present, the lubricant of a motor car is not taken with the importance of the case, causing consumers to give greater importance to the price of the same rather than quality, and components. Research conducted an analysis of oils through a tribological test in a gasoline engine, with the aim of knowing the wear on internal engine components, based on the damning limits and their established ranges. Two brands of SAE 20W50 API SN lubricant, was used to perform a comparative table between them and under the same conditions. Among the results, it was observed that the oil A has a higher percentage of soot generated $0.02 \%$ higher than $\mathrm{B}$, for reasons of lower viscosity at high temperature. A substantial difference in amount of oxidation by a value greater than $27 \%$ in oil $\mathrm{B}$, found what specifically affects the life of the lubricant and jeopardizes 
the protection of engine components. The amounts of $\mathrm{Cu}, \mathrm{Si}$ and $\mathrm{Al}$ were within the limits damning both the lubricant and lubricant B was no substantial difference is these products. Instead Na values were outside the range ( 23 and $24 \mathrm{ppm}$ ) for coolant leaks. It is disclosed then that despite having the same specification between different brands, you can generate different wears due to internal components and characteristics of each oil type.

Key words: tribology; engine wear; oils; lubricants

\section{Introducción}

La tribología estudia la ciencia y tecnología relacionadas a la lubricación, fricción y el desgaste de materiales en partes móviles o estacionarias. El estudio siempre estará relacionado al funcionamiento de máquinas de forma general. (Linares, 2013). El análisis tribológico se lo realiza comúnmente en motores de combustión interna de ciclo Otto, diésel, o en motores estacionarios para conocer el tipo de desgaste a los que estos están sometidos, y dependiendo del lubricante utilizado.

Otro campo en el cual se realiza este análisis es en el parque automotor, en este se lo efectúa comúnmente en flotas de vehículos, ya que de esta manera el control del mantenimiento preventivo del vehículo tiende a ser más eficiente. En el mercado existe una variedad de lubricantes, los que ofrecen diferentes tipos de protección para el motor, esta protección dependerá de la base y constitución que el lubricante esté fabricado, así como los diferentes aditivos y detergentes que este contenga.

Con el fin de conocer si el lubricante utilizado y el desgaste interno del motor es el adecuado, según las especificaciones del fabricante, se realizará un análisis en un vehículo de prueba, el cual recorrerá un mismo kilometraje pero con diferentes tipos de lubricantes, así obtendremos los datos de los porcentajes de desgaste interno del motor y concluimos si parte de los lubricantes que encontramos en el mercado son los adecuados para la lubricación del motor.

Cerca del 30\% de la energía que se pierde en la industria mundial, se debe a la fricción, así países como Inglaterra, Japón y Alemania pierden anualmente más de 200 millones de dólares como resultado del desgaste." Martínez Pérez (párr.4).

La tribología es una ciencia necesaria para la exposición detallada de los procesos no reversibles que tienen lugar en la mecánica en cuanto a la interacción continua de las superficies bajo las fuerzas de rozamiento, y que contribuyen a explicar los fenómenos de la perdida de energía y materiales en esa interacción. (Martínez Pérez; 2011).

En la actualidad existen varios tipos de análisis dentro de la tribología, de los cuales los más conocidos son:

Ferrografía directa (conteo de partículas).- Se basa en una medición cuantitativa de la concentración de las partículas ferrosas en una muestra de fluido (generalmente aceite) a través de la precipitación particular a través de un tubo de vidrio insertado en un fuerte campo magnético. 
Espectrofotometría de absorción atómica (detección de elementos presentes).- Se basa en un análisis y conteo de partículas presentes en el fluido, es el análisis más conocido para la implementación de programas de mantenimiento predictivo, debido a que en los centros de mantenimiento e industriales, los proveedores de lubricante y servicios ofrecen esta asistencia como un valor agregado o servicio profesional.

Una de las aplicaciones más conocidas se da en el análisis del desgaste a través del aceite lubricador en motores de combustión interna. Los motores constan de innumerables elementos y cada componente del mismo está diseñado y construido con diversos materiales, y cada uno de sus componentes trabaja a diversas solicitaciones, por ello el desgaste puede provenir de varias partes del motor. En la tabla 1 se puede observar el origen probable de los materiales que reporta un análisis.

Tabla 1. Origen de materiales

\begin{tabular}{|c|c|c|c|c|c|c|c|c|c|}
\hline Motor & $\mathrm{Fe}$ & $\mathrm{Cu}$ & $\mathrm{Pb}$ & $\mathrm{Al}$ & $\mathrm{Si}$ & $\mathrm{Cr}$ & $\mathrm{Sn}$ & $\mathrm{Na}$ & $\mathrm{K}$ \\
\hline Cojinetes & & $\mathrm{x}$ & $\mathrm{x}$ & $\mathrm{x}$ & & & $\mathrm{x}$ & & \\
\hline Bujes & & $\mathrm{x}$ & & $\mathrm{x}$ & & & $\mathrm{x}$ & & \\
\hline Árbol de levas & $\mathrm{x}$ & & & & & & & & \\
\hline Refrigerante & & & & & $\mathrm{x}$ & $\mathrm{x}$ & & $\mathrm{x}$ & $\mathrm{x}$ \\
\hline Cigüeñal & $\mathrm{x}$ & & & & & & & & \\
\hline Camisa & $\mathrm{x}$ & & & & & $\mathrm{x}$ & & & \\
\hline Válvula de escape & $\mathrm{x}$ & & & & & $\mathrm{x}$ & & & \\
\hline Cojinetes anti-fricción & $\mathrm{x}$ & & & & & $\mathrm{x}$ & & & \\
\hline Empaques & & & & & $\mathrm{x}$ & & & & \\
\hline Gasolina & & & $\mathrm{x}$ & & & & & $\mathrm{x}$ & \\
\hline Carcasa & $\mathrm{x}$ & & & $\mathrm{x}$ & & & & & \\
\hline Tierra & & & & & $\mathrm{x}$ & & & & \\
\hline Aditivo & & & & & $\mathrm{x}$ & & & & \\
\hline Enfriador de aceite & & $\mathrm{x}$ & & & & & & & \\
\hline Bujes de bomba de aceite & & & & $\mathrm{x}$ & & & & & \\
\hline Bomba de aceite & $\mathrm{x}$ & & & $\mathrm{x}$ & & & & & \\
\hline Pistones & $\mathrm{x}$ & & & $\mathrm{x}$ & & & & & \\
\hline Anillos & $\mathrm{x}$ & & & & & $\mathrm{x}$ & & & \\
\hline Volandas de empuje & & $\mathrm{x}$ & $\mathrm{x}$ & $\mathrm{x}$ & & & $\mathrm{x}$ & & \\
\hline Engranajes de cadenilla & $\mathrm{x}$ & & & & & & & & \\
\hline Turbo & $\mathrm{x}$ & & & $\mathrm{x}$ & & & & & \\
\hline Guías de válvulas & $\mathrm{x}$ & $\mathrm{x}$ & & & & & & & \\
\hline Tren de válvulas & $\mathrm{x}$ & & & & & & & & \\
\hline Bujes de bielas & & $\mathrm{x}$ & $\mathrm{x}$ & $\mathrm{x}$ & & & & $\mathrm{x}$ & \\
\hline Bielas & $\mathrm{x}$ & & & & & & & & \\
\hline
\end{tabular}

Fuente: (Widman internacional, 2015) 
Para determinar si el desgate se encuentra dentro de los rangos permitidos es necesario basarse en ciertos parámetros preestablecidos denominados límites condenatorios. Los límites condenatorios son los valores normales que generalmente deben tener un motor en cuanto a desgastes de material se refiere. Estos nos indican el estado en el cual el motor puede estar internamente, cabe recalcar que entre menor sea el numero o grado de desgaste, este será favorable para la vida útil del motor. Widman International (2015) publicó en su página una tabla con los valores o límites aceptables de desgastes de materiales (Tabla 2).

Tabla 2. Límites condenatorios por material

\begin{tabular}{ccccc}
\hline Parámetro & $\begin{array}{c}\text { Valor } \\
\text { mínimo }\end{array}$ & $\begin{array}{c}\text { Valor } \\
\text { medio }\end{array}$ & $\begin{array}{c}\text { Valor } \\
\text { máximo }\end{array}$ & Unidad \\
\hline $\mathrm{Cr}$ & 0 & 0 & 10 & $\mathrm{Ppm}$ \\
$\mathrm{Cu}$ & 0 & 2 & 50 & $\mathrm{Ppm}$ \\
$\mathrm{Fe}$ & 0 & 1 & 75 & $\mathrm{Ppm}$ \\
$\mathrm{Al}$ & 0 & 0 & 50 & $\mathrm{Ppm}$ \\
$\mathrm{Mg}$ & 0 & 0 & 5 & $\mathrm{Ppm}$ \\
$\mathrm{Na}$ & 0 & 0 & 75 & $\mathrm{Ppm}$ \\
$\mathrm{Ni}$ & 0 & 0 & 5 & $\mathrm{Ppm}$ \\
$\mathrm{Pb}$ & 0 & 0 & 20 & $\mathrm{Ppm}$ \\
$\mathrm{Si}$ & 0 & 4 & 20 & $\mathrm{Ppm}$ \\
$\mathrm{Sn}$ & 0 & 0 & 10 & $\mathrm{Ppm}$ \\
$\mathrm{Sb}$ & 0 & 0 & 5 & $\mathrm{Ppm}$ \\
$\mathrm{Zn}$ & 0 & 0 & 5 & $\mathrm{Ppm}$ \\
$\mathrm{B}$ & 0 & 0 & 5 & $\mathrm{Ppm}$ \\
$\mathrm{Ag}$ & 0 & 0 & 5 & $\mathrm{Ppm}$ \\
$\mathrm{Mn}$ & 0 & 0 & 5 & \\
\hline & 0 & 0 & 5 & 5 \\
\hline
\end{tabular}

Fuente: (Widman international, 2015)

Otro análisis generalmente incluido en este tipo de reportes es el de propiedades fisicoquímicas. Incluye referencias a la oxidación, apariencia, \% agua y viscosidad.

Tabla 3. Análisis de propiedades físico químicas del lubricante

\begin{tabular}{ccccc}
\hline Propiedad & Oxidación & Apariencia & $\begin{array}{c}\text { Contenido de } \\
\text { H2O \% }\end{array}$ & Viscosidad \\
\hline Valor & 0.00 & Clara brillante & 0.00 & 45.1 \\
Estado & Normal & Normal & Normal & Normal \\
\hline
\end{tabular}


Fuente: (Widman internacional, 2015)

Los reportes se lo hace tomando en cuenta el origen del material y su límite condenatorio, por ejemplo si existiera un valor superior a $75 \mathrm{ppm}$ de $\mathrm{Na}$, el reporte estaría orientado a la revisión de alguna fuga del líquido refrigerante a través de algún empaque deteriorado.

\section{Materiales y Métodos}

En Ecuador, son pocas las empresas que realizan un análisis previo al lubricante que se va a utilizar en los motores, es por ello que actualmente existe una fomentación sobre una cultura de análisis hacia los diferentes tipos de lubricantes, ya que no solo depende de una marca comercial, sino de los diferentes componentes del lubricante.

El estudio es de tipo exploratorio, en donde mediante una prueba de ruta se efectuó un análisis tribológico de un motor de combustión interna de ciclo Otto, utilizando dos tipos de lubricantes, la ruta trazada fue utilizada en los dos procesos de análisis, con el fin de poder realizar una comparación del desgaste de las partes internas de un motor según el lubricante utilizado.

En la figura 1 se observa el vehículo Suzuki Forza2 de año de fabricación 1999, con un kilometraje de $297784.8 \mathrm{~km}$, de $993 \mathrm{~cm} 3$ de cilindrada, que fue utilizado para la prueba de ruta con combustible Extra de 89 octanos.

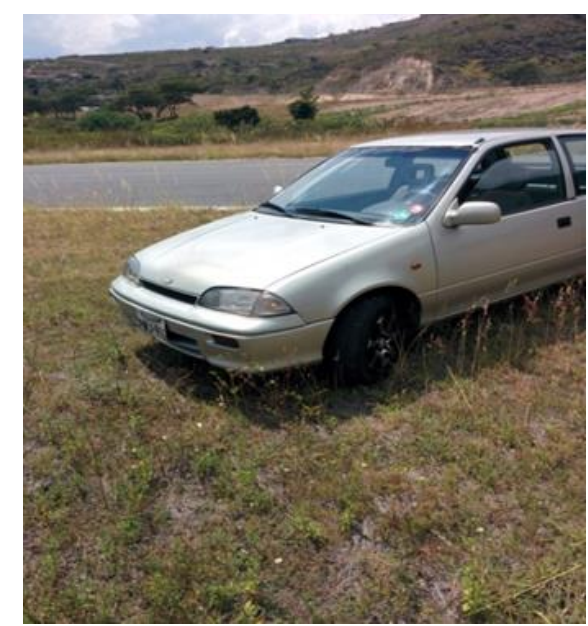

Figura 1. Vehículo Suzuki Forza2 (vehículo de prueba)

El kilometraje de prueba a recorrer en el vehículo fue de $1380.7 \mathrm{KM}$, kilometraje que fue trazado en diferentes rutas las cuales se incluyen a continuación.

Una de las rutas utilizadas era el traslado desde el taller donde se efectuaba el mantenimiento del vehículo hacia el domicilio del mismo. La ruta fue de 26.1 KM de recorrido, la misma se realizó en un total de 18 veces, en un horario en el cual el tráfico vehicular era fluido permitiéndonos realizar el recorrido en un tiempo aproximado de 35 minutos, y manteniendo una velocidad promedio de $75 \mathrm{Km} / \mathrm{h}$. 
En esta ruta se acumuló un total de 469.8 km, y un aproximado de 630 minutos de trabajo del motor.

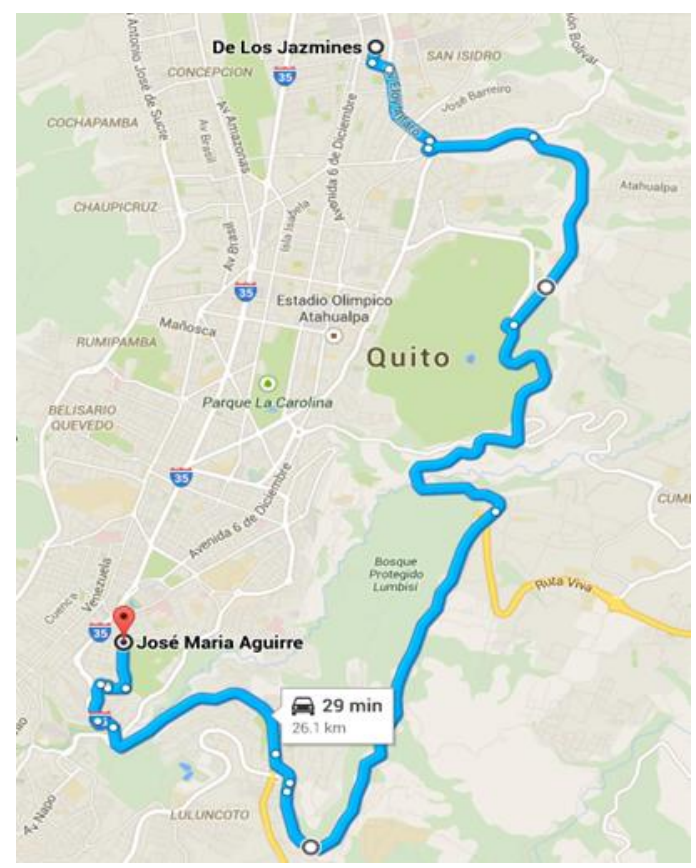

Figura 2. Ruta taller - domicilio

La segunda etapa trazada brindó diferente tipos de cambios climáticos, los que afectarían al funcionamiento y rendimiento del vehículo, esto debido a la variación de altura a la cual se encontraba el vehículo. En la etapa 2, el vehículo recorrió un total de $354 \mathrm{Km}$ en total, la ruta trazada fue Quito-Yahuarcocha; Yahuarcocha-Quito; en la cual se acumuló un total de 354 $\mathrm{Km}$. Y un aproximado de 450 minutos de funcionamiento 


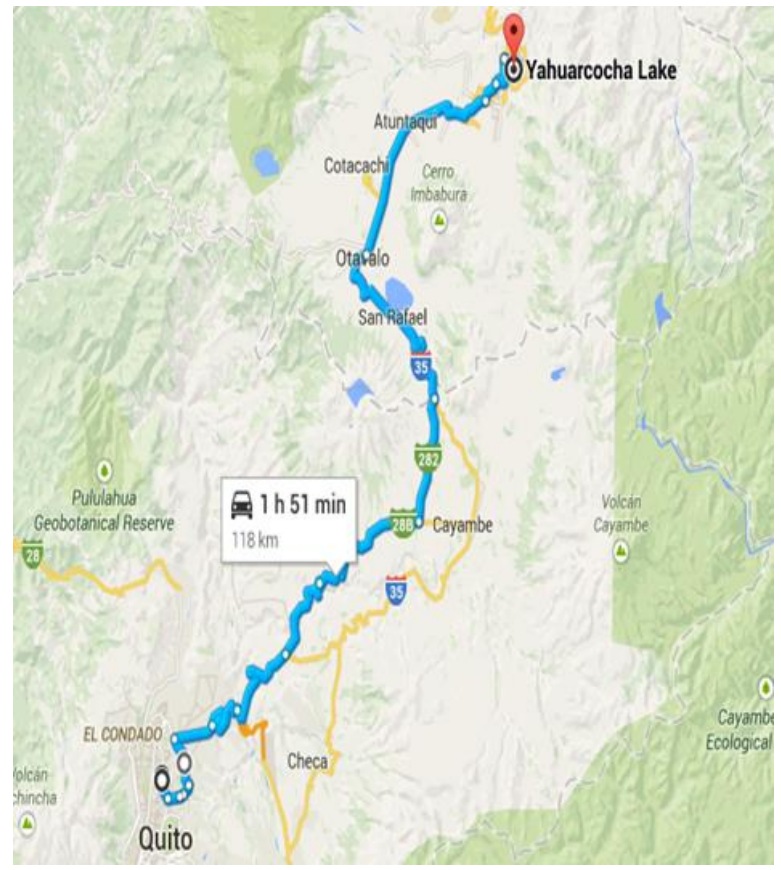

Figura 3. Ruta Quito-Yahuarcocha

De la misma manera la ruta trazada en su mayor porcentaje se la hizo evitando lugares de alto tráfico vehicular, ya que el propósito de la ruta es tener condiciones similares para las diferentes pruebas y un motor en constante funcionamiento a un alto régimen de trabajo, para obtener mejores resultados relacionados al desgaste dentro del motor.

Se utilizó la pista del autódromo " José Tobar Tobar" ubicado en la ciudad de Ibarra, el cual consta con $3.7 \mathrm{Km}$ de recorrido, en este lugar se pudo realizar pruebas controladas del vehículo, efectuando siempre un funcionamiento de alto régimen, y exponiendo al vehículo a altas temperaturas, para de esta manera poder exigir al lubricante a que realice su trabajo.

Se realizó un aproximado de 150 vueltas en total acumulando un kilometraje de 556.2 $\mathrm{km}$, cabe indicar que las exigencias que el motor sufrió en este proceso fueron muy grandes, con revoluciones superiores a las $4000 \mathrm{rpm}$.

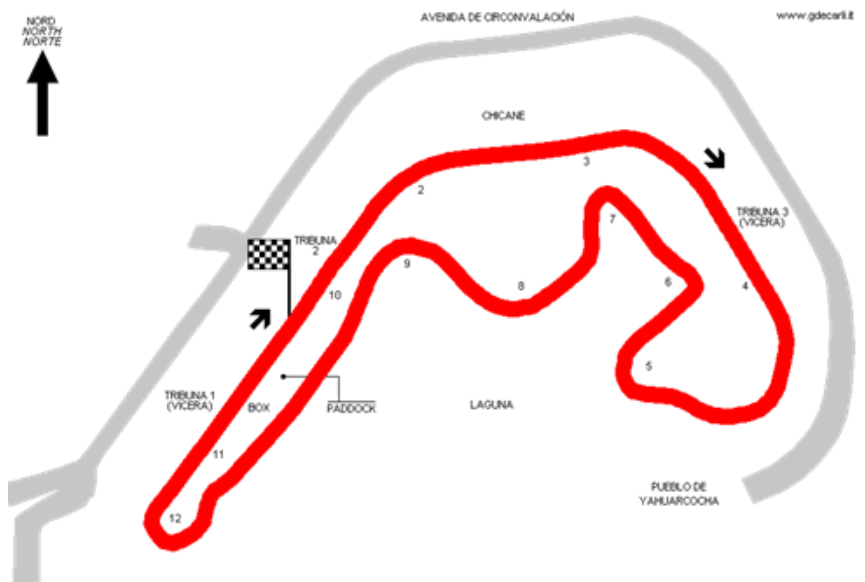

Figura 4. Autódromo internacional José Tobar-Tobar 
Se realizó un número programado de arranques en frio, ya que se conoce que el mayor porcentaje de desgaste interno del motor se produce en los arranques en frio. Para poder realizar los mismos fue necesario dejar reposar al vehículo aproximadamente 1 hora como mínimo, para que de esta manera el vehículo tome una temperatura relativamente baja y no cercana a la de trabajo, de la misma manera con esta acción el aceite llega a reposar en su totalidad en el cárter del vehículo.

Se tomó en cuenta el número de arranques en caliente, se los realizó aproximadamente con un tiempo de 10 minutos de descanso del motor, tiempo necesario para que el aceite llegue a reposar en su totalidad en el cárter. En esta situación el aceite no desciende a temperaturas bajas de trabajo. Esto se hizo con el fin de exigir al motor y aceite a sufrir un mayor desgaste, y de esta manera obtener una mayor cantidad de datos en el análisis.

Se realizó un control al vehículo en cuanto a kilometraje recorrido, numero de arranques en frio, arranques en caliente, tiempos de reposo y horas aproximadas de uso (Tabla 4).

\section{Tabla 4. Control de trabajo del vehículo}

\begin{tabular}{|c|c|c|}
\hline \multicolumn{3}{|c|}{ Vueltas en pista } \\
\hline Km acumulado & Tiempo acumulado & Cantidad de vueltas \\
\hline $556,02 \mathrm{Km}$ & 360,66 Minutos & 150 \\
\hline Velocidad promedio & $\begin{array}{c}\text { Tiempo promedio por } \\
\text { vuelta } \\
\end{array}$ & $\begin{array}{c}\text { Descanso por número } \\
\text { de vueltas } \\
\end{array}$ \\
\hline 92,5 KM/H & 156,20 (segundos) & 1 por cada 15 vueltas \\
\hline \multicolumn{3}{|c|}{ Ruta en carretera, autopista y ciudad } \\
\hline Km acumulado & Tiempo acumulado & Velocidad promedio \\
\hline 824,68 & 1080 MINUTOS & $45,6 \mathrm{KM} / \mathrm{H}$ \\
\hline \multicolumn{3}{|c|}{ Arranques en frio } \\
\hline Cantidad & $\begin{array}{c}\text { Tiempo de reposo del } \\
\text { lubricante }\end{array}$ & $\begin{array}{l}\text { Promedio de arranques } \\
\text { por km. }\end{array}$ \\
\hline 13 & 45 - 60 MINUTOS & 106,20 \\
\hline \multicolumn{3}{|c|}{ Arranques en caliente } \\
\hline Cantidad & $\begin{array}{c}\text { Tiempo de reposo del } \\
\text { lubricante }\end{array}$ & $\begin{array}{l}\text { Promedio de arranques } \\
\text { por km. }\end{array}$ \\
\hline 48 & 7 - 15 MINUTOS & 28,76 \\
\hline
\end{tabular}

Una vez realizadas las tres rutas trazadas, y en lo posible en los mismos tiempos, kilometrajes y condiciones, se procedió a cambiar de aceite. De esta manera a futuro al realizar el análisis de aceites, se tiene una comparación muy aproximada y bajo similares condiciones en cuanto al desgaste interno del motor.

Alcanzado el kilometraje deseado se procedió a extraer el lubricante utilizado, para proceder a la obtención del análisis, esto se realizó mediante los siguientes procesos.

Proceso 1.- cambio de lubricante del motor a temperatura de trabajo, durante el drenado del lubricante por medio del tapón del cárter se obtiene la muestra de aceite con el uso de un 
embudo limpio y desinfectado, en un envase de $120 \mathrm{ml}$ según la especificación del laboratorio para posteriormente realizar el análisis.

Proceso 2.- sellado de la muestra y se procede a llenar la ficha para su posterior envió y análisis.

Recolectada la primera muestra se procede a realizar las rutas con el segundo lubricante, cabe recalcar que las rutas son las mismas para ambos lubricantes, esto con el fin de poder realizar una comparación de los lubricantes. El primer lubricante utilizado, se lo denominó Lubricante A y el segundo, Lubricante B, para lo que se utilizó sus respectivas fichas técnicas proporcionado por cada empresa representante oficial del lubricante en Ecuador.

Tabla 5. Ficha técnica de lubricante A

\begin{tabular}{ccc}
\hline ESPECIFICACIONES & \multicolumn{2}{c}{ SAE 20W50, API SN } \\
\hline PRUEBA & UNIDAD & VALOR TIPICO \\
\hline Viscosidad @ 100 C & CSt & 18,09 \\
Viscosidad @ 40 C & CSt & 159,1 \\
Índice de viscosidad & & 126 \\
Viscosidad CCS @ -15 C & cP Max & 9.500 \\
Viscosidad de bombeo @ -20 C & cP Max & 60.000 \\
Viscosidad HTHS & cP Min & 3,7 \\
Punto de escurrimiento & C Max & -33 \\
Punto de inflamación PMCC & C min & $>200$ \\
Densidad relativa @ 15C & & 0,884 \\
\hline
\end{tabular}

Fuente: (Datos del fabricante)

Tabla 6. Ficha técnica de lubricante B

\begin{tabular}{lll}
\hline ESPECIFICACIONES & \multicolumn{2}{l}{ SAE 20W50, API SN } \\
\hline PRUEBA & UNIDAD & VALOR TIPICO \\
\hline Viscosidad @ 100 C & CSt & 12 \\
Viscosidad @ 40 C & CSt & 150 \\
Índice de viscosidad & & 139 \\
Viscosidad CCS @ -15 C & cP Max & 7.100 \\
Viscosidad de bombeo @ -20 C & cP Max & 34.000 \\
Viscosidad HTHS & cP Min & 4,85 \\
Punto de escurrimiento & C Max & -34 \\
Punto de inflamación PMCC & C min & 242
\end{tabular}


TBN

Cenizas sulfatadas

$0,84 \%$

\section{Fuente: (Datos del fabricante)}

\section{Resultados y Discusión}

Se determinó la curva de viscosidad de los lubricantes A y B (Figura 5), con los datos obtenidos del lubricante en cuanto a la viscosidad a $100{ }^{\circ} \mathrm{C}$ y $40{ }^{\circ} \mathrm{C}$, y mediante el uso de la página oficial de Widman International, de esta manera se pudo generar las curvas a diferentes temperaturas.

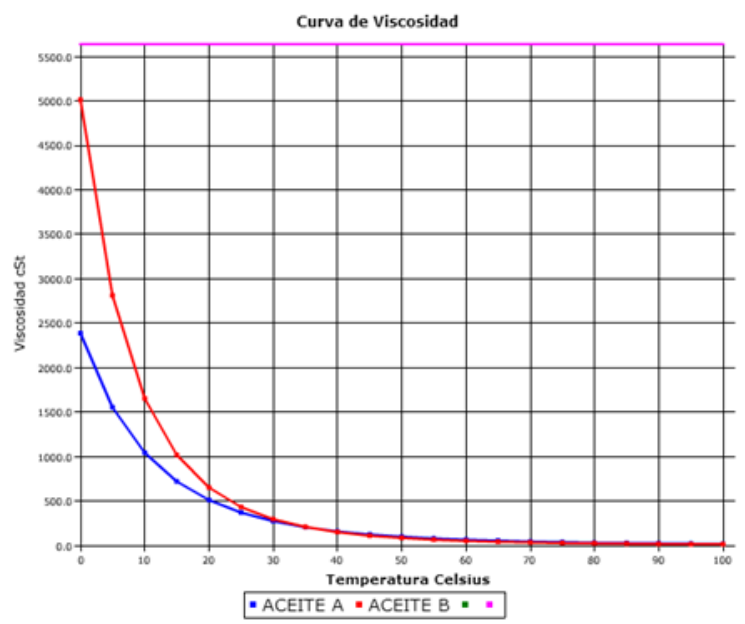

Figura 5. Viscosidad Vs. Temperatura lubricantes A y B

A menores temperaturas el aceite A trabajaría a menor viscosidad lo que es un buen indicativo respecto a desgaste inicial antes de la temperatura de trabajo del motor.

\section{Tabla 7. Resultado de análisis Aceite A}

\begin{tabular}{cc}
\hline Aceite A & \\
\hline Hollín & $3,80 \%$ \\
Oxidación & NEGATIVA \\
Sulfatación & NEGATIVA \\
Viscosidad a $100 \mathrm{c}$ & $16,2 \mathrm{CST}$ \\
Viscosidad a $40 \mathrm{c}$ & $139,84 \mathrm{CST}$ \\
TBN & \\
IV & 116 \\
Agua & NEGATIVO \\
Combustible & POSITIVO \\
Punto de inflamación & $215 \mathrm{C}$ \\
$\mathrm{Cu}$ & $3 \mathrm{ppm}$ \\
$\mathrm{Fe}$ & $12 \mathrm{ppm}$ \\
$\mathrm{Cr}$ & $0 \mathrm{ppm}$ \\
$\mathrm{Pb}$ & $0 \mathrm{ppm}$ \\
$\mathrm{Si}$ & $5 \mathrm{ppm}$
\end{tabular}




\begin{tabular}{cc}
$\mathrm{Al}$ & $7 \mathrm{ppm}$ \\
$\mathrm{Na}$ & $23 \mathrm{ppm}$ \\
$\mathrm{Ca}$ & 0,15 \\
$\mathrm{Zn}$ & 0,06 \\
\hline
\end{tabular}

Tabla 8. Resultado de análisis Aceite B

\begin{tabular}{cc}
\hline Aceite B & \\
\hline Hollín & $3,60 \%$ \\
Oxidación & $27 \%$ \\
Sulfatación & NEGATIVO \\
Viscosidad a $100 \mathrm{c}$ & $15,18 \mathrm{CST}$ \\
Viscosidad a $40 \mathrm{c}$ & $133,7 \mathrm{CST}$ \\
TBN & \\
IV & 116 \\
Agua & NEGATIVO \\
Combustible & POSITIVO \\
Punto de inflamación & $194 \mathrm{C}$ \\
$\mathrm{Cu}$ & $2 \mathrm{ppm}$ \\
$\mathrm{Fe}$ & $13 \mathrm{ppm}$ \\
$\mathrm{Cr}$ & $0 \mathrm{ppm}$ \\
$\mathrm{Pb}$ & $0 \mathrm{ppm}$ \\
$\mathrm{Si}$ & $6 \mathrm{ppm}$ \\
$\mathrm{Al}$ & $5 \mathrm{ppm}$ \\
$\mathrm{Na}$ & $24 \mathrm{ppm}$ \\
$\mathrm{Ca}$ & 0,17 \\
$\mathrm{Zn}$ & 0,05 \\
&
\end{tabular}

Tabla 9. Comparativa por límites condenatorios

\begin{tabular}{llll}
\hline & Aceite A & Aceite B & Límites Condenatorios \\
\hline $\mathrm{Cu}$ & $3 \mathrm{ppm}$ & $2 \mathrm{ppm}$ & $2-15 \mathrm{ppm}$ \\
$\mathrm{Fe}$ & $12 \mathrm{ppm}$ & $13 \mathrm{ppm}$ & $2-50 \mathrm{ppm}$ \\
$\mathrm{Cr}$ & $0 \mathrm{ppm}$ & $0 \mathrm{ppm}$ & $1-8 \mathrm{ppm}$ \\
$\mathrm{Pb}$ & $0 \mathrm{ppm}$ & $0 \mathrm{ppm}$ & $2-10 \mathrm{ppm}$ \\
$\mathrm{Si}$ & $5 \mathrm{ppm}$ & $6 \mathrm{ppm}$ & $2-10 \mathrm{ppm}$ \\
$\mathrm{Al}$ & $7 \mathrm{ppm}$ & $5 \mathrm{ppm}$ & $2-15 \mathrm{ppm}$ \\
$\mathrm{Na}$ & $23 \mathrm{ppm}$ & $24 \mathrm{ppm}$ & $0-10 \mathrm{ppm}$ \\
\hline
\end{tabular}

Se pueden encontrar anomalías de desgaste o malfuncionamiento de motor por el diferencial superior de los límites condenatorios en un valor superior de Sodio, la razón viene dada por el consumo de refrigerante y daño interno del sello de los empaques. 
Gráficas de los materiales de mayor impacto según análisis

\section{Lubricante A-B (Hollín)}

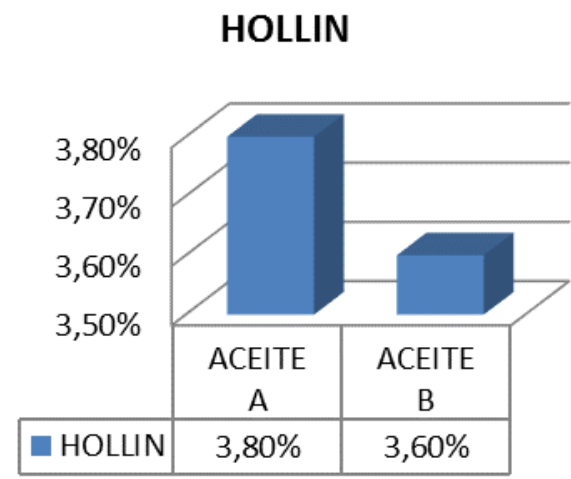

Grafica 1. Resultado hollín

El hollín se presenta debido al aceite combustionado, esto indica que los rines que sellan al pistón con el cilindro no realizan un trabajo adecuado y el sello de gases puede estar comprometido, de esta manera el acceso de los gases de la cámara de combustión al cárter afectará al aceite en su composición. En los resultados se observa que el aceite A tiene mayor porcentaje de hollín (relativamente mínimo), la razón para que este fenómeno se dé, es por la viscosidad del aceite a baja y alta temperatura. El aceite al ser menos viscoso pasa con mayor facilidad hacia la cámara de combustión. Es necesario mencionar que debido al kilometraje y desgaste del vehículo, este genera un consumo de aceite entre mantenimientos.

\section{Oxidación Lubricante A-B}

\section{OXIDACIÓN}

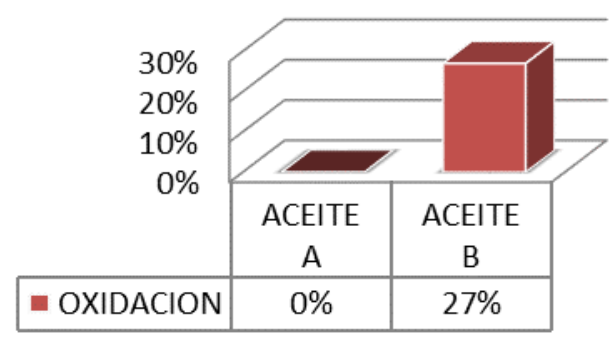

Grafica 2. Resultado oxidación

La oxidación se origina por cambios químicos en los aditivos del lubricante combinado con las altas temperaturas de trabajo. En las pruebas realizadas las exigencias que sufrió el motor fueron altas, prueba de ello fue la variación del nivel del líquido refrigerante, de esta manera se pudo concluir que existió un sobrecalentamiento del motor produciendo un consumo de líquido refrigerante. La variación de resultados entre los lubricantes se da específicamente por la diferencia de antioxidantes que existen en el lubricante, por ende el aceite B contiene menor 
cantidad de este tipo de aditivo, resultando una mayor cantidad de oxidación, que puede afectar específicamente a la vida útil del lubricante del motor.

\section{Lubricante A-B (Cu)}

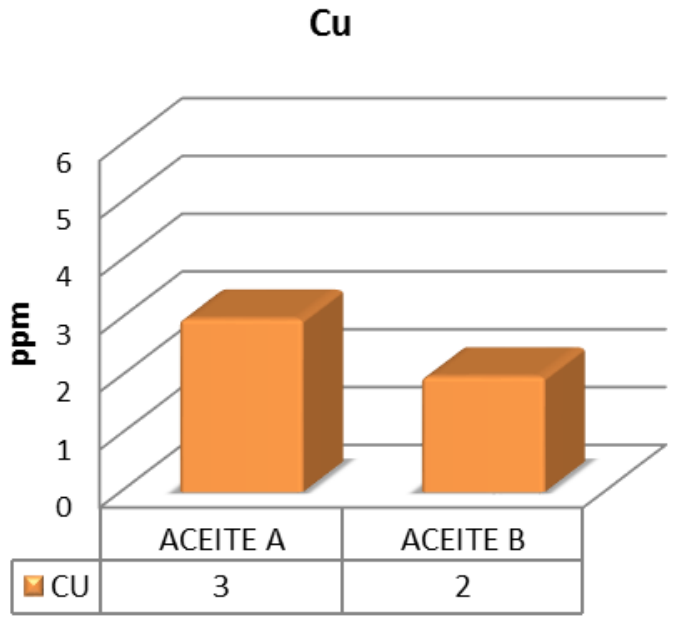

Grafica 3. Resultado Cu

Se encontró desgaste de cobre, que está relacionado generalmente a bujes y cojinetes. Estos elementos normalmente están aleados y estructuralmente poseen capas superficiales de diferentes metales blandos requeridos y diseñados para disminuir el desgaste y absorber el impacto. Generalmente este desgaste es normal puesto que los metales blandos evitan un deterioro superior de las bielas y el cigüeñal. Los resultados tanto del aceite A como del aceite B estuvieron dentro de los límites condenatorios normales. Se produce este tipo de desgaste por el deterioro de la viscosidad en el aceite, producto de su uso; esto da como consecuencia la disminución de la lubricación hidrodinámica necesaria para proveer la lubricación límite.

\section{Lubricante A-B (Fe)}

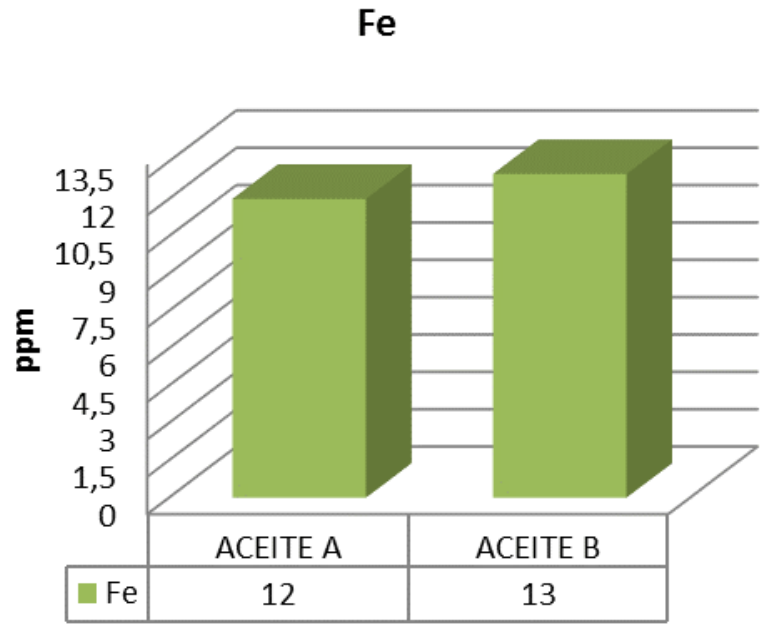

Grafica 4. Resultado Fe 
Se determinó que tanto el aceite A como el aceite B están dentro de los límites normales, y el valor de diferencia es mínimo. La presencia de hierro $(\mathrm{Fe})$ en el aceite tiene origen en la producción de oxidación de ciertos elementos o su desgaste por fricción como es el caso de los cilindros y segmentos. Los índices de oxidación pueden incrementar dependiendo de la presencia de otros elementos como el agua originada por alguna fuga. De la misma manera el desgaste puede incrementarse por la entrada en exceso de silicio por tierra, en el caso de filtros dañados, condiciones de trabajo del motor o aceite contaminado con partículas que rocen las paredes.

\section{Lubricante A-B (Cr)}

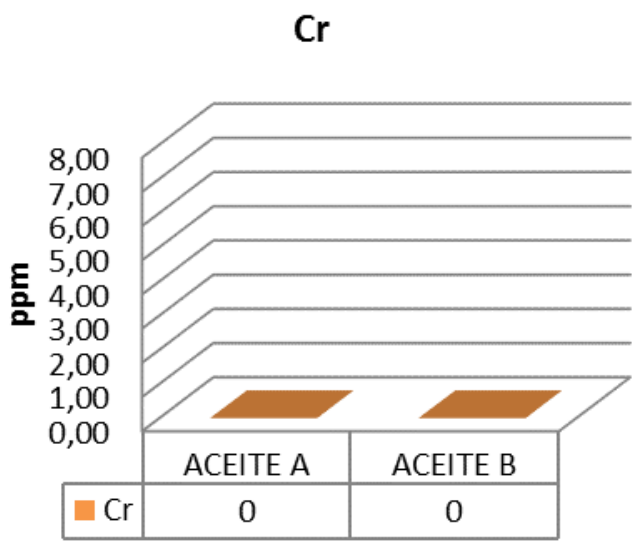

Grafica 5. Resultado Cr

En el análisis la cantidad de cromo fue nula, así se conoce que dentro del motor no existe desgaste de válvulas. Esto debido a que el cromo es característico para aleaciones principales en válvulas de admisión y escape así como componentes en pasadores de biela (bulones) o camisas. La aparición de cromo en resultados de laboratorio es un indicador de suciedad o impurezas en el aceite.

\section{Lubricante A-B (Pb)}

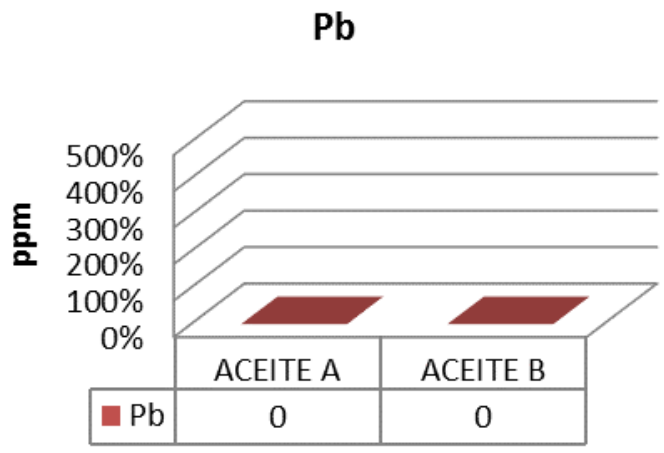

Grafica 6. Resultado Pb 
Se demostró que la cantidad de plomo es nula, siendo indicativo de dos causas: la primera es que no existe consumo o fuga de combustible en exceso y la segunda es que no se ha producido un desgaste significativo en los cojinetes. Se debe considerar también que el kilometraje recorrido no es tan amplio como para tener desgastes significativos de plomo. La presencia de elementos corrosivos en el aceite, la baja cantidad de aditivos anticorrosivos y condiciones de trabajo (inclusive el clima) puede generar malos resultados en diferentes análisis.

\section{Lubricante A-B (Si)}

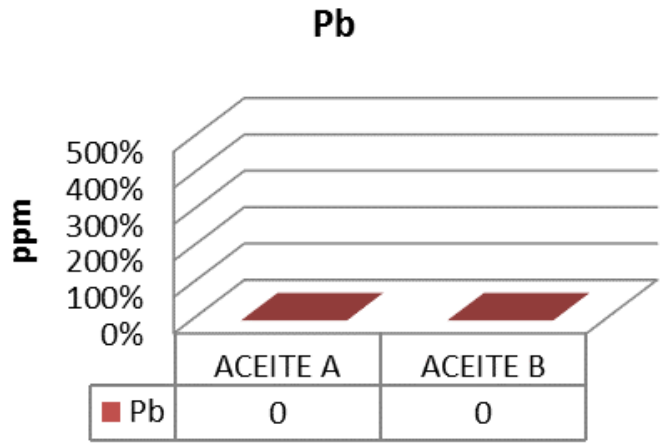

Grafica 7. Resultado Si

Se demuestra que los valores se encuentran dentro de los límites permitidos, tanto en el lubricante $\mathrm{A}$ como en el lubricante $\mathrm{B}$, la diferencia de estos no es significativa. Comúnmente el exceso de silicio puede darse por un mal funcionamiento de los filtros de aire, los que permiten la entrada de tierra. También algunos refrigerantes pueden contener silicio, inclusive varios lubricantes dentro de sus aditivos pueden contener este elemento.

\section{Lubricante A-B (Al)}

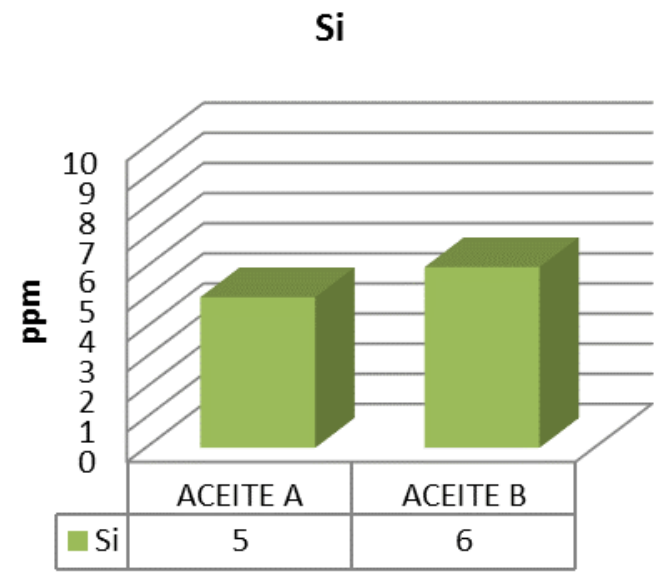

Grafica 8. Resultado Al

Los valores resultantes mostraron que los dos lubricantes se encuentran dentro de los límites condenatorios, pero tenemos una mayor cantidad de desgaste en el aceite A, esto se debe a que genera menor lubricación hidrodinámica y por ende produce mayor desgaste en cojinetes y 
bujes. La lubricación hidrodinámica inferior provoca un desgaste directo en cojinetes, bujes y en ciertas ocasiones en pistones provocado por el cascabeleo excesivo.

\section{Lubricante A-B (Na)}

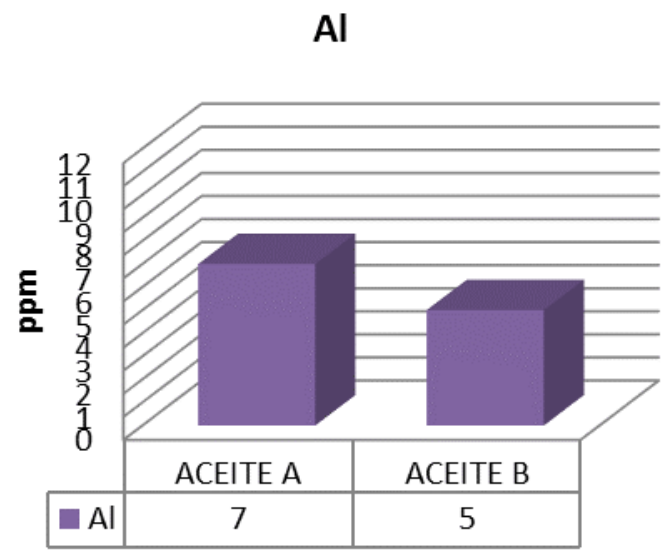

Grafica 9. Resultado Na

El sodio $(\mathrm{Na})$ es principalmente un componente de refrigerantes y ciertas aleaciones en bujes, en este análisis se observa que los valores obtenidos tanto en el lubricante A como en el B están fuera de los límites; esto revela que es una consecuencia que en el vehículo existe un consumo y fuga de líquido refrigerante.

\section{Conclusiones}

A pesar de tener una misma especificación entre diferentes marcas, se pueden generar diferentes degastes debido a la cantidad de componentes internos y características aditivas de cada tipo de aceite.

Entre los resultados se observó que el aceite A generaba mayor porcentaje de hollín: 0,02\% superior al B, esto se da a causa de una viscosidad inferior a alta temperatura. Se encontró una sustancial diferencia, en cantidad de oxidación por un valor superior al $27 \%$ en el aceite B, lo que afecta específicamente a la vida útil del lubricante y pone en riesgo la protección de los componentes del motor. Las cantidades de $\mathrm{Cu}$, Si y Al se encontraban dentro de los límites condenatorios aceptables tanto en el lubricante A como en el lubricante B, la diferencia no fue sustancial es estos productos.

Los valores de Sodio $(\mathrm{Na})$ estuvieron fuera del rango por fugas de líquido refrigerante. El desgaste de los componentes varía según las condiciones de trabajo a las cuales este expuesto, siendo mayores los desgastes al estar el motor a temperaturas inferiores a las de trabajo. El desgaste de los diferentes componentes está ligado específicamente a la lubricación hidrodinámica y a la lubricación límite, la cual se da dependiendo de los aditivos que cada uno de los lubricantes posee.

\section{Bibliografía}


Aguirre, Viviana (2008). Estudio experimental de la fricción pistón-camisa en un motor de combustión interna

http://www.amaq.com/tutoriales/Tutorial\%20de\%20Ferrografia\%20A-MAQ\%202005.pdf

Albarracín, A.P.: Tribología y Lubricación Industrial y Automotriz. Medellín, Litocha 2da Ed. 2000.

Alonso, J.M. (2007). Técnicas del Automóvil. 10ma edición Paraninfo España

API ORG. $\quad$ (2015); $\quad$ Publications; $\quad$ Recuperado 18/03/2015.http://www.api.org/ /media/files/certification/engine-oildiesel/publications/engine-oil-guide_spanish-3q2012.pdf?la=en.

Bilbao, Jesús; Málaga, Adolfo (2014) Lubricación y mantenimiento de motores; Recuperado 03/04/2015 http://lubrication-management.com/wpcontent/uploads/sites/3/2014/07/Lubricaci\%C3\%B3n_motores_gas_ES.pdf

De Castro, Miguel Vicente (1995). Trucaje de motores de 4 tiempos. Grupo editorial CEAC España

Gil, Hermógenes (2000).Manual práctico del automóvil. Cultural Madrid-España

Linares, Omar O. Tribología Y Mantenimiento Proactivo, Widman International S.R.L. Santa Cruz, Bolivia

Stefano Gillieri (2005). Preparación de motores de serie para competición. Grupo editorial CEAC España

Widman International (2015); boletines informativos: pdf 19; recuperado 12/03/2015. http://widman.biz/boletines_informativos/19.pdf 